\title{
Philosophiques
}

\section{La flèche brisée}

\section{Ronan de Calan}

Volume 37, numéro 2, automne 2010

URI : https://id.erudit.org/iderudit/045194ar

DOI : https://doi.org/10.7202/045194ar

Aller au sommaire du numéro

Éditeur(s)

Société de philosophie du Québec

ISSN

0316-2923 (imprimé)

1492-1391 (numérique)

Découvrir la revue

Citer ce document

de Calan, R. (2010). La flèche brisée. Philosophiques, 37(2), 479-482.

https://doi.org/10.7202/045194ar

Ce document est protégé par la loi sur le droit d'auteur. L'utilisation des services d'Érudit (y compris la reproduction) est assujettie à sa politique d'utilisation que vous pouvez consulter en ligne.

https://apropos.erudit.org/fr/usagers/politique-dutilisation/
Cet article est diffusé et préservé par Érudit.

Érudit est un consortium interuniversitaire sans but lucratif composé de l’Université de Montréal, l'Université Laval et l'Université du Québec à Montréal. Il a pour mission la promotion et la valorisation de la recherche. https://www.erudit.org/fr/ 


\title{
La flèche brisée
}

\author{
RONAN DE CALAN
}

IHPST, Paris I

Quand on sait le soin que Jocelyn Benoist prend à toute chose, on ne peut pas ne pas prendre au sérieux la couverture de son dernier ouvrage, un tableau de Paul Klee datant de 1922 et qui a pour titre Betroffener Ort, littéralement: "Lieu visé ». La flèche de l'intentionalité s'y trouve brisée, ou plutôt déviée, réfractée lors de son passage dans un contexte qui prend la forme d'une strate de milieux homogènes aux teintes de plus en plus sombres à mesure qu'on approche du monde sublunaire dont le sol, lui, est tout à fait opaque. Ce que cette flèche brisée vise, en outre, n'est pas un objet, mais un lieu, le «lieu visé " précisément, ou plutôt le lieu atteint après déviation, le but requalifié. Ce lieu concret et non abstrait (ce n'est pas l'espace de la géométrie) consiste en un ensemble de relations ou d'états de chose qui lient moins des objets que des formes, des êtres en mouvement et mêmes des intentions (figurées par des flèches qui supportent quelque chose comme des portées musicales sur lesquelles sont placées, en guise de notes, des spirales, équivalents de la représentation de la parole dans l'iconographie médiévale).

Betroffener Ort se veut l'illustration de la thèse que Jocelyn Benoist étaye sur plus de trois cents pages d'analyses de cas: celle du "réalisme intentionnel». Le réalisme intentionnel figure en quelque sorte l'Aufhebung, le dépassement d'une contradiction non médiatisée entre un point de vue «externe» en philosophie, dont le point d'aboutissement est le béhaviorisme et ses avatars (le génial Willard Van Orman Quine est sans aucun doute le représentant le plus remarquable de cette perspective), et un point de vue «interne» qui culmine avec les doctrines sartriennes du tout intentionnel ou néo-hégéliennes (chez Descombes ou chez McDowell par exemple, bien que dans des perspectives différentes) de l'intentionalité du monde. En un certain sens, la psychologie comme la philosophie de la connaissance n'ont fait que répéter depuis bientôt un siècle l'équivalent d'une plaisanterie en vogue dans l'actuelle Russie: de même qu'un chevelu succède toujours à un chauve et un chauve à un chevelu à la présidence, depuis la formation de l'URSS - Lénine puis Staline, Khrouchtchev puis Brejnev, Gorbatchev puis Eltsine, Poutine puis Medvedev, Poutine puis Medvedev, Poutine puis Medvedev, etc. et à l'infini - de même, un béhavioriste succède toujours à un intentionnaliste, les variantes n'étant que des sophistications du modèle initial.

Comme toute thèse révolutionnaire en philosophie, la thèse $\mathrm{du}$ « réalisme intentionnel» identifie le socle théorique commun aux deux doctrines en affrontement permanent, l'accord implicite que recouvre le désaccord explicite, et elle propose une échappée. Ce socle commun, quel est-il ? D'abord, une élimination du contexte, c'est-à-dire de l'extériorité nécessaire 
à la qualification de l'acte comme intentionnel (soit par dissolution de l'intentionalité elle-même au profit d'une extériorité radicale, soit par dilution du contexte dans un faisceau de visée). En effet, pour que l'acte puisse être qualifié d'intentionnel, il doit être orienté vers quelque chose d'extérieur à l'acte lui-même. Mais si cette extériorité ne désigne que ce sur quoi on a prise, elle n'a aucune prégnance. En revanche, si tout est extériorité, comme le veut le béhavioriste radical, c'est la nature de l'acte qui se perd puisque celui-ci devient lui-même effet. Une extériorité sans frontière est alors tout aussi dépourvue de sens. Autre propriété de ce socle: un recentrement des ontologies qui supportent la description des actes, supprimant ou mobilisant l'intentionalité, sur la catégorie de l'objet. Ces deux arguments, qui composent aussi les deux axes de ce repère commun, peuvent être articulés si l'on estime, avec Jocelyn Benoist, que l'une et l'autre perspectives font bel et bien litière de quelque chose qu'on appelle le réel. Plus exactement, les béhavioristes comme les intentionnalistes ravalent le réel à la catégorie de la res, de la chose matérielle, et se privent alors de la possibilité de rendre compte de la manière dont on compose avec lui sans se dissoudre en lui ou sans le dissoudre lui-même.

Aussi la première thèse forte défendue par Jocelyn Benoist est-elle nécessairement une thèse pascalienne: l'intentionalité est bornée de toute part par du non-intentionnel.

Un fétu d'intentionalité dans un univers fondamentalement non intentionnel, telle est, on l'aura compris, la représentation du monde, pascalienne, mais d'un Pascal sans Dieu en quelque sorte, vers laquelle on tendrait. Représentation fort banale, dira-t-on. Ce qui est somme toute normal, puisqu'elle est vraie. Mais la pointe d'une telle représentation est que, selon elle, non seulement l'intentionnel est toujours cerné par le non-intentionnel, mais celui-ci pénètre profondément celui-là, est toujours immiscé en lui, de façon à introduire en lui une forme d'équivocité ontologique: la possibilité structurelle de sa dérive vers le non-intentionnel ${ }^{1}$.

Ce qui qualifie le réel alors n'est pas seulement son indifférence à l'acte, c'est dans une certaine mesure sa résistance, c'est-à-dire aussi sa capacité à requalifier l'acte lui-même (à dévier la flèche), voire à l'annuler (la briser). Mais déployer une telle efficacité, le réel ne doit pas être simplement composé de choses matérielles, si possible inertes, réductible comme chez Descartes à l'étendue, ni d'objet, c'est-à-dire de choses sur lesquelles on a prise.

La seconde thèse qui s'en déduit, en quelque sorte, est la suivante: un acte intentionnel est non seulement un acte borné, mais aussi et surtout un acte normé. Une catégorie d'acte a effectivement un contexte qui correspond aussi et surtout à l'ensemble des circonstances typiques qu'il s'assigne ou se

1. J. Benoist, Sens et sensibilité. L'intentionalité en contexte, Paris, Le Cerf, 2009, p. 173. 
prescrit dans sa réalisation. Les circonstances d'un acte de langage diffèrent des circonstances d'un acte purement physique, par exemple un mouvement volontaire. Ces actes sont alors normés par des contraintes qu'ils convoquent nécessairement: interlocuteurs (absence d'interlocuteurs), ou lieu, etc., pour l'acte de langage, espace disponible, gravité, etc., pour le mouvement volontaire. Ce sont ces circonstances typiques qui vont décider de la pertinence ou de la non-pertinence de l'acte, c'est-à-dire de son efficacité, de sa capacité à atteindre son but, quitte à en changer. Par exemple, le fait d'énoncer une phrase comme "la guerre est déclarée " sur une scène de théâtre, dans un amphithéâtre ou dans l'hémicycle d'une assemblée ou, pour prendre un cas plus intéressant, le fait d'énoncer cette même phrase sur une scène de théâtre construite dans l'hémicycle d'une assemblée lors d'une cérémonie spéciale, donne non seulement un effet perlocutoire mais un sens différent à l'acte lui-même. Autrement dit, le réel est, dans sa résistance même, fait des conventions que convoquent et colportent nos actes eux-mêmes, et des effets induits de ces conventions non seulement sur la pertinence mais sur l'orientation de nos actes, voire sur leur qualité d'actes. Ainsi, ils peuvent être destitués, par les normes mêmes qu'ils suscitent, de leur supposée intentionalité (c'est le cas de la déclaration de guerre proclamée sur la scène de théâtre, y compris dans l'hémicycle d'une assemblée). Le réel, irréductible à la catégorie de la res, est aussi conventionnel, sans être pour autant le produit d'un accord explicite: la texture conventionnelle du réel n'est évidemment pas tissée par un contrat passé entre plusieurs individus (c'est la base d'une vision juridique, par exemple de la sociologie), mais par la typicité de nos actes eux-mêmes.

Ce qui nous conduit à une troisième thèse: un acte intentionnel, quelque normé qu'il soit et contraint par ces normes, n'est pas condamné à se briser, à s'annuler au moindre obstacle. Comme un corps élastique, il peut fléchir, ce qui signifie que je puis en cours d'acte changer d'intention. C'est précisément là plus qu'ailleurs, dans ces accidents de l'intentionalité, que je puis identifier cette propriété particulière de la visée, à savoir d'être libre tout en étant à la fois bornée et normée. C'est le but requalifié du tableau de Paul Klee: même déviée, la flèche touche un but, qui n'était pas forcément le but initial, mais qui le devient par la force des choses. Cette liberté a naturellement une contrepartie: tout acte intentionnel ne peut se délester d'une forme d'ambiguïté intrinsèque, une ambiguité ontologique qui tient à la fois à ce principe d'utilisation multiple d'une seule action, que Jocelyn Benoist emprunte à Marcel Proust, mais aussi et également, du point de vue théorique, au principe corrélatif d'interprétation multiple de l'intentionalité ou de la non-intentionalité de l'acte. Par exemple, ce qui pour moi peut être un «but requalifié » désignera aux yeux d'autrui un acte non intentionnel ou un échec de la visée, et inversement.

Les trois thèses ici défendues se ramènent donc dans une certaine mesure à trois contraintes que le réel impose à l'intentionalité comme 
relation fondamentale (et, dans une certaine mesure, définition de la pensée): la borne (ou le bornage), la norme et l'ambiguïté. Il est fascinant de constater qu'avant Jocelyn Benoist ce travail de limitation de l'intentionalité par le réel n'avait jamais sérieusement été envisagé, en tous les cas sous une forme articulée, comme un triple effet de structure: on était contraint, soit à abandonner tout principe de réalité (c'est-à-dire de contrainte) — et force est de reconnaître qu'à l'exception d'une psychologie ou d'une sociologie du reste périmées, la philosophie est la seule à avoir emprunté cette voie -, soit à se débarrasser purement et simplement de l'intentionalité elle-même - c'était l'option sans doute moins contestable de la linguistique, de la sociologie et de l'anthropologie dans leur époque structuraliste. La tactique de Sens et sensibilité consiste à arracher l'intentionalité à son arrière-plan mentaliste et à l'intégrer dans une théorie générale de la pratique en utilisant massivement les références pragmatistes. Cela signifie aussi que pour Jocelyn Benoist, le modèle sémantique qu'il avait pu utiliser par ailleurs ne suffit plus à pallier ses défauts. Il s'agit au fond d'un mouvement analogue à celui que Marx engageait dans les Thèses sur Feuerbach concernant la vision matérialiste du réel, réduit justement à une pure extériorité, celle de la res, celle des choses: «Le défaut principal, jusqu'ici, de tous les matérialistes (y compris celui de Feuerbach) est que l'objet, la réalité, la sensibilité, ne sont conçus que sous la forme de l'objet ou de l'intuition, non comme activité humaine sensible, pratique, non comme subjective ${ }^{2}$.» J. Benoist rejoint la praxis marxiste en occupant une position symétrique sur ses principaux enjeux. Vous avez dit: révolution?

2. Karl Marx, thèses sur Feuerbach, trad. G. Badia (modifiée), Paris, Éditions sociales, 1968 , p. 31. 